hep-th/yymmnnn

\title{
Low-energy effective theory for a Randall-Sundrum scenario with a moving bulk brane
}

\author{
Ludovica Cotta-Ramusino*, ${ }^{*}$ and David Wands* \\ * Institute of Cosmology and Gravitation, University of Portsmouth, Portsmouth, P01 2EG, \\ United Kingdom \\ ${ }^{\dagger}$ Laboratory for Computation and Visualization in Mathematics and Mechanics, EPFL FSB \\ IMB, Ecole Polytechnique Fédérale de Lausanne, CH-1015, Switzerland.
}

\begin{abstract}
We derive the low-energy effective theory of gravity for a generalized RandallSundrum scenario, allowing for a third self-gravitating brane to live in the 5D bulk spacetime. At zero order the 5D spacetime is composed of two slices of anti-de Sitter spacetime, each with a different curvature scale, and the 5D Weyl tensor vanishes. Two boundary branes are at the fixed points of the orbifold whereas the third brane is free to move in the bulk. At first order, the third brane breaks the otherwise continuous evolution of the projection of the Weyl tensor normal to the branes. We derive a junction condition for the projected Weyl tensor across the bulk brane, and combining this constraint with the junction condition for the extrinsic curvature tensor, allows us to derive the first-order field equations on the middle brane. The effective theory is a generalized Brans-Dicke theory with two scalar fields. This is conformally equivalent to Einstein gravity and two scalar fields, minimally coupled to the geometry, but nonminimally coupled to matter on the three branes.
\end{abstract}

\section{Introduction}

The possibility that our Universe might be a $(3+1)$-dimensional membrane (brane) embedded in some higher $(4+n)$-dimensional spacetime (bulk), as suggested by M-theory [1], has been extensively studied over recent years. In brane models, although gravity can propagate in the whole bulk, other matter fields are localized on the brane. In particular, Randall and Sundrum [2, 3] proposed a brane-world model in which we live on a 4D brane embedded in a $5 D$ anti-de Sitter (AdS) spacetime. In 2 two branes are placed at the fixed points $(y=0$ and $y=y_{0}$ ) of an orbifold $\mathbf{S}^{\mathbf{1}} / \mathbf{Z}_{\mathbf{2}}$, where we identify $y \rightarrow-y$ and $y-y_{0} \rightarrow y_{0}-y$, and $y$ is the extra-coordinate. The bulk AdS spacetime can be then thought of as being bounded by the two $4 D$ branes. The induced metrics on the branes can be flat (Minkowski) if a fine tuning condition is imposed on the vacuum energies or tensions of the branes:

$$
\sigma_{0}=-\sigma_{y_{0}}=\frac{6}{\kappa_{5}^{2}} \frac{1}{\ell}
$$

where $\sigma_{0}$ and $\sigma_{y_{0}}$ are the tensions on the hidden brane at $y=0$ at on the visible brane at $y=y_{0}, \kappa_{5}^{2}$ is the $(5 D)$ gravitational coupling and $\ell$ is the AdS curvature scale. 
Assuming the bulk metric obeys the 5D vacuum Einstein equations, then the projected gravitational field equations on the brane are modified with respect to general relativity [4]. Two additional terms appear with respect to general relativity: a local term, quadratic in the energy-momentum tensor on the brane, and a non-local term, which is a projection of the $5 D$ Weyl tensor, namely $E_{\alpha \beta}$.

The contribution to the $4 D$ effective theory of $E_{\alpha \beta}$, which describes the contribution of the bulk gravitational field on the brane and influences the brane cosmological evolution, is of crucial importance [5]. Although the quadratic source term becomes relevant only at high energies, the projected Weyl tensor may remain non-negligible in the low-energy regime, where one would hope to recover general relativity.

The projected Weyl tensor generally has non-closed equations on the brane [4, 5] and in general one should solve the full bulk gravitational field equations. However it is possible to derive a scheme which allows one to self-consistently solve the $5 D$ Einstein equations in the low-energy regime, and carefully construct the projected Weyl tensor on the brane.

A low-energy perturbation scheme was proposed in [6] for the Randall-Sundrum (RS) two brane scenario [2]. (See also [7].) The low-energy regime is defined as the regime in which the matter energy density on the brane is much smaller than the RS brane tension (1.1). The perturbation parameter is defined as the ratio between these two energy densities, and the $5 D$ Einstein equations can be solved at different orders in the perturbation parameter. This method allows one in principle to derive the effective Einstein equations on the brane at each order, although of particular interest is the first order correction, which is the most relevant at low energies.

In the original derivation by Kanno and Soda [6], the full $5 D$ equations of motion were solved at each order in the bulk by performing a perturbation expansion in the metric. In the alternative derivation by Shiromizu and Koyama [8], the expansion was rather done directly in terms of the extrinsic curvature and the projected Weyl tensor, whose equations of motion can then be solved in the bulk at each order. Crucially, the use of the junction conditions enables one to express the Weyl tensor as a function of the matter content of the branes and the physical distance between the branes, interpreted as the radion field. This finally allows for the derivation of the effective Einstein equations induced on the brane at low energy. On both branes, at first order, the final effective theory is called by the authors of [6, 8] a quasi-scalar-tensor gravity theory, where the Brans-Dicke field couples through different gravitational coupling constants with matter on each brane.

The low-energy effective actions on the negative and positive brane respectively are [6, 9]

$$
\begin{aligned}
S_{-} & =\frac{\ell}{2 \kappa_{5}^{2}} \int d^{4} x \sqrt{-g}\left[\Phi R-\frac{\omega_{-}(\Phi)}{\Phi}(\nabla \Phi)^{2}\right] \\
& +\int d^{4} x \sqrt{-g}\left(\mathcal{L}_{-}+(1+\Phi)^{2} \mathcal{L}_{+}\right)
\end{aligned}
$$

where $\omega_{-}(\Phi)=-3 \Phi[2(\Phi+1)]^{-1}$, and

$$
\begin{aligned}
S_{+} & =\frac{\ell}{2 \kappa_{5}^{2}} \int d^{4} x \sqrt{-\hat{g}}\left[\Psi \hat{R}-\frac{\omega_{+}(\Psi)}{\Psi}(\hat{\nabla} \Psi)^{2}\right] \\
& +\int d^{4} x \sqrt{-\hat{g}}\left(\mathcal{L}_{+}+(1-\Psi)^{2} \mathcal{L}_{-}\right)
\end{aligned}
$$


where $\omega_{+}(\Psi)=3 \Psi[2(1-\Psi)]^{-1}$, and $\mathcal{L}_{-}$and $\mathcal{L}_{+}$are the Lagrange densities for matter fields on the branes.

The theories, as expected for Brans-Dicke theories, are conformally equivalent to Einstein gravity plus a minimally-coupled scalar field, described by

$$
\begin{aligned}
S_{E F} & =\frac{\ell}{2 \kappa_{5}^{2}} \int d^{4} x \sqrt{-\tilde{g}}\left[\tilde{R}-(\tilde{\nabla} \chi)^{2}\right] \\
& +\int d^{4} x \sqrt{-\tilde{g}}\left[\sinh ^{4}(\chi / \sqrt{6}) \mathcal{L}_{-}+\cosh ^{4}(\chi / \sqrt{6}) \mathcal{L}_{+}\right]
\end{aligned}
$$

where the conformal factors $\Omega^{2}$ (negative brane) and $\hat{\Omega}^{2}$ (positive brane) are

$$
\Omega^{2}=\frac{1}{\sinh ^{2}(\chi / \sqrt{6})} \quad \hat{\Omega}^{2}=\frac{1}{\cosh ^{2}(\chi / \sqrt{6})}
$$

and $\sqrt{(1+\Phi)}=\operatorname{coth}(\chi / \sqrt{6})$ and $\sqrt{(1-\Psi)}=\tanh (\chi / \sqrt{6})$, where $\Psi<1$ [10, 11, 12].

There are many additional complications to take into account if one wishes to relate simple brane-world models to realistic configurations in the context of superstring and Mtheory. In any of brane-world models it is important to derive the low-energy effective theory on the $4 D$ branes. In this paper we consider just the effect of adding an additional brane in the bulk. We will focus on a generalization of [8] to a three brane scenario. The work presented in the paper was originally presented in [12]. Since then a number of other authors [13, 14, 15] have investigated the low-energy effective theory corresponding to three branes in an AdS bulk, in particular considering the effective potential for D-branes in warped flux compactifications [13] and a simple geometrical model for brane inflation [14]. A generalized Randall-Sundrum scenario with three branes was also previously studied in [10, 16], and multi-brane collisions were considered in [17.

The plan of the paper is the following. In the second section we derive the low-energy effective theory in a generalized Randall- Sundrum two-branes scenario, allowing for a third brane to live in the bulk. In section (2.1) we discuss the preliminaries, adapting the covariant formalism of [8] to a three brane model. In section (2.2) we discuss the background solution and (2.3) we derive the effective Einstein equations at first order on the third brane. The effective theory turns out to be a Brans-Dicke theory, with two independent scalar fields, one minimally coupled with the geometry. Finally in section (2.4) we show that a conformal transformation relates the effective theory on the third brane to Einstein gravity plus two minimally coupled scalar fields. In the third and final section we draw our conclusions.

\section{First order effective theory at the third brane}

In this section we derive the low-energy effective theory at first order in a three brane scenario, using the perturbative scheme introduced in Ref. [6]. We choose to follow the covariant approach as in Ref. [8].

\subsection{Preliminaries}

We consider an extension of the Randall-Sundrum two brane model [2]. The three branes are separated by slices of AdS bulk, each characterized by a different curvature scale, as is 


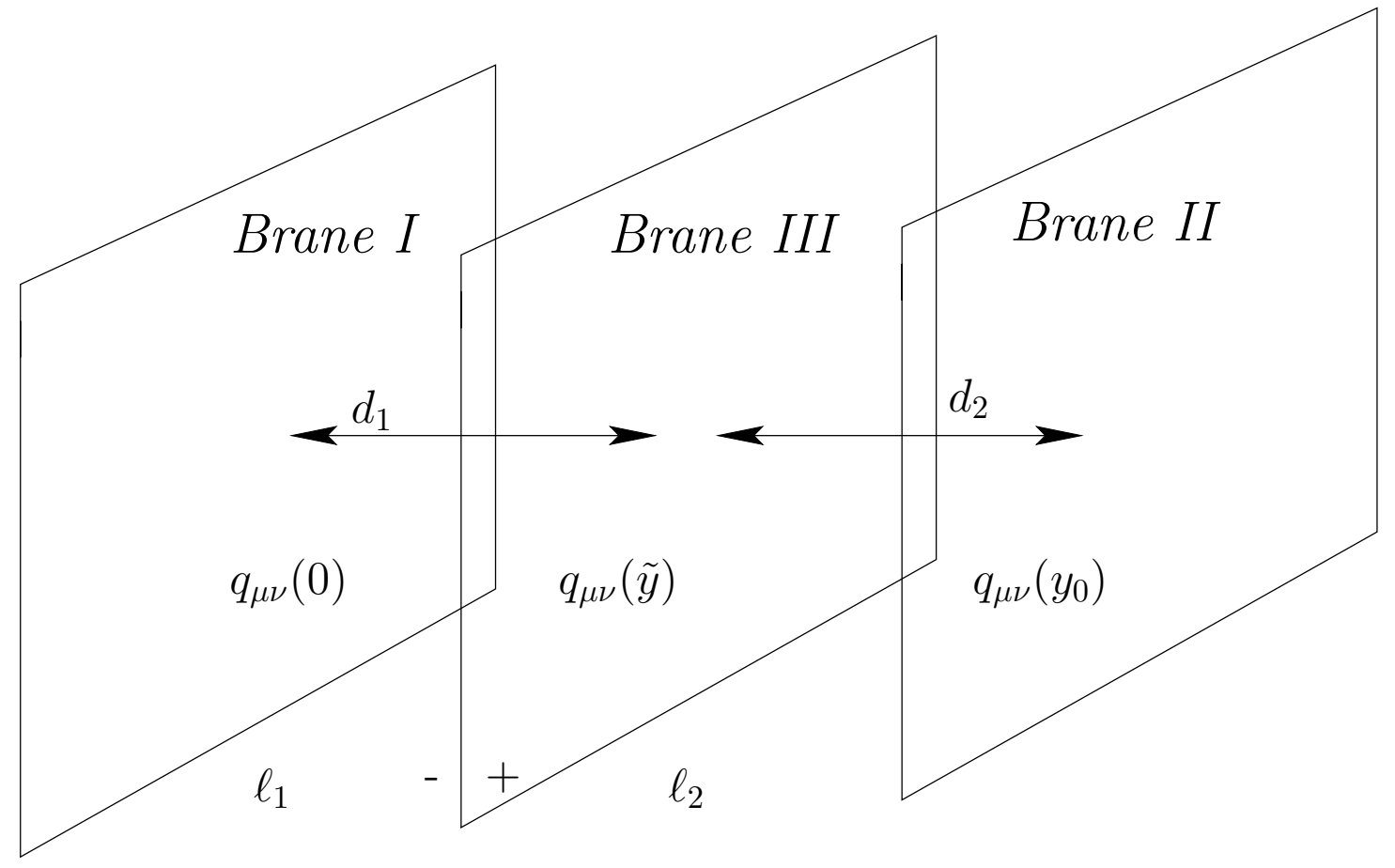

Figure 1: The branes are separated by slices of AdS $5 D$ spaces, each with a different curvature scale, $\ell$. The sign + and - are placed either side of the third brane to show our convention for the orientation of the normal vector field. The induced metrics, $q_{\mu \nu}$, on the three branes are conformally rescaled by the geometrical warp factor.

schematically shown in figure 1. Brane I and II are still at the fixed points of the $\mathbf{S}_{\mathbf{1}} / \mathbf{Z}_{\mathbf{2}}$ orbifold, and therefore they respect a $Z_{2}$ symmetry, wheareas Brane III is not at a fixed point of the orbifold. Two natural scalar degrees of freedom characterize this scenario: one associated with the overall distance between the two boundary branes, one associated with the relative position of the third bulk brane.

The Randall-Sundrum metric [2] reads

$$
d s^{2}=e^{2 \phi(y, x)} d y^{2}+q_{\alpha \beta}(y, x) d x^{\alpha} d x^{\beta},
$$

where $q_{\alpha \beta}$ is the metric induced on the brane. The normal vector field $n^{\alpha}$ is chosen to have the same orientation throughout the bulk, so that locally, at each brane, it is pointing in the same direction and $q_{\alpha \beta}=q_{\alpha \beta}^{5 D}-n_{\alpha} n_{\beta}$ if $q_{\alpha \beta}^{5 D}$ is the $5 D$ metric. The proper distances between the branes are defined via

$$
d_{1}(x)=\int_{0}^{\tilde{y}} e^{\phi\left(y^{\prime}, x\right)} d y^{\prime}, \quad d_{2}(x)=\int_{\tilde{y}}^{y_{0}} e^{\phi\left(y^{\prime}, x\right)} d y^{\prime} .
$$

Our objective is to derive at first order the effective Einstein equations on the third (i.e. middle) brane: this will be enough to determine the effective Einstein equations on the other two branes, as they are related to the Einstein equations on the third brane by an appropriate conformal transformation, given the specific form of the metric (2.6) which in turn implies 
that the background metrics on the three branes can be conformally transformed into each other.

In the perturbative scheme introduced in Ref. [6], the low-energy regime is defined as the regime in which the energy densities on the branes are negligible with respect to the brane tension,

$$
\rho_{i} \ll\left|\sigma_{i}\right|,
$$

or, taking into account (1.1) and $8 \pi G_{(4)}=\kappa_{5}^{2} \ell^{-1}$ where $G_{(4)}$ is the effective $4 D$ Newton's constant 4,

$$
\left(\frac{\ell}{L}\right)^{2} \ll 1
$$

where $\ell$ is the bulk curvature scale in the AdS slice and $L$ is the brane curvature scale. Therefore the background solution is the vacuum spacetime, and perturbations are introduced as matter is added on the branes. The parameter of expansion is given by [6]

$$
\epsilon=\left(\frac{\ell}{L}\right)^{2}
$$

and, accordingly, expansions of the extrinsic curvature

$$
K_{\alpha}^{\beta} \equiv \frac{1}{2} q^{\beta \sigma} \mathcal{L}_{n} q_{\alpha \sigma}=\frac{1}{2} q^{\beta \sigma} e^{-\phi} \partial_{y} q_{\alpha \sigma},
$$

and the projected 5D Weyl tensor,

$$
E_{\alpha}^{\beta} \equiv C_{\rho \alpha \sigma}^{\beta} n^{\rho} n^{\sigma}
$$

around the vacuum solutions can be considered as follows [8]

$$
\begin{aligned}
K_{\alpha}^{\beta} & =K_{\alpha}^{\beta(0)}+\epsilon K_{\alpha}^{\beta(1)}+\epsilon^{2} K_{\alpha}^{\beta(2)}+\ldots \\
E_{\alpha}^{\beta} & =\epsilon E_{\alpha}^{\beta(1)}+\epsilon^{2} E_{\alpha}^{\beta(2)}+\ldots
\end{aligned}
$$

where $E_{\alpha}^{\beta(0)}=0$ for the Randall-Sundrum brane-world. With the expansions (2.13) and (2.14), the evolution equations for $K_{\alpha}^{\beta}$ and $E_{\alpha}^{\beta}$ can be solved at different orders, and subsequentlty, so can the induced Einstein equations on the brane [6] [8]. In particular the evolution equations in the bulk for $K_{\alpha}^{\beta}$ and $E_{\alpha}^{\beta}$ are given by the Lie derivatives along $n^{\alpha}$, as these describe the changes of both the tensors along the integral curves of the normal vector field $n^{\alpha}$.

At zero order for three branes, i.e. for the background solution, the equations are no more complicated than in the two brane case. The bulk solution between each pair of branes corresponds to a region of anti-de Sitter, with curvature scales $\ell_{1}>\ell_{2}$, and the Weyl tensor vanishes at this order. In each region the extrinsic curvature tensor is constant but has two different values in the two different slices of AdS spaces (giving rise to a constraint on the tension of the bulk brane).

At first order, some further steps are required, the reason being mainly that the third brane breaks the otherwise continuous evolution of the Weyl tensor in the bulk. Briefly, the plan for deriving the first-order effective theory is the following 
- Junction conditions

Write the junction conditions for each of the three branes. In particular the third brane $(y=\tilde{y})$ is not at a fixed point of the orbifold so that we have to include the jump suffered by the extrinsic curvature tensor at the passage through brane III.

\section{- Evolution equations}

Write the evolution equations at first order (i.e. the Lie derivatives at first order) for $E_{\alpha}^{\beta}$ and $K_{\alpha}^{\beta}$ which are the same as in the two brane scenario. However the firstorder solutions to these equations now hold only separately in the two AdS regions of spacetime, so special attention is required at brane III where in general the solutions are discontinuous. In particular, from the Lie derivative of $K_{\alpha}^{\beta}(\tilde{y})$, we write the junction condition at the third middle brane as a function of the source terms, the tensor $E_{\alpha}^{\beta}$ at both sides of brane III ( + and - as sketched in figure 1) and the kinetic terms associated with the proper distances $d_{1}$ and $d_{2}$ (2.7).

- Consistency of Einstein equations on brane III

Impose the requirement of consistency of the induced Einstein equations on the third brane. This leads to a junction condition for $E_{\alpha}^{\beta}$ at this brane.

- Weyl tensor

Obtain a system of two independent equations in the two unknowns $E_{\alpha}^{\beta}(\tilde{y})^{+}$and $E_{\alpha}^{\beta}(\tilde{y})^{-}$, from the previous steps. The system is then solved for $E_{\alpha}^{\beta}(\tilde{y})^{+}$(analogously it could be solved for $\left.E_{\alpha}^{\beta}(\tilde{y})^{-}\right)$. We then obtain the Weyl tensor on one side of brane III as a function of the sources and the kinetic terms associated with both $d_{1}$ and $d_{2}$ (2.7).

\section{- Einstein equations}

Finally, substituting the expressions for $K_{\alpha}^{\beta}(\tilde{y})^{+}$and $E_{\alpha}^{\beta}(\tilde{y})^{+}$in equation [8]

$$
\left.G_{\alpha}^{\beta}\right|_{\tilde{y}} ^{+}=\left[-\left.\frac{2}{\ell_{2}}\left[K_{\alpha}^{\beta}-\delta_{\alpha}^{\beta} K\right]\right|_{\tilde{y}} ^{+}-\left.E_{\alpha}^{\beta}\right|_{\tilde{y}} ^{+}\right]
$$

enables us to obtain the Einstein equations on brane III.

We show that the effective gravity theory obtained at first-order is a generalized Brans-Dicke theory with two scalar fields.

\subsection{Junction conditions and background solution}

If the direction of the normal vector field $n^{\alpha}$ to a brane is chosen to be the same throughout the bulk, the junction conditions read

- brane I

$$
\left.\left(K_{\alpha}^{\beta}-\delta_{\alpha}^{\beta} K\right)\right|_{0}=-\frac{\kappa_{5}^{2}}{2}\left(-\sigma_{0} \delta_{\alpha}^{\beta}+\left.T_{\alpha}^{\beta}\right|_{0}\right)
$$

- brane III

$$
\left.\left[K_{\alpha}^{\beta}-\delta_{\alpha}^{\beta} K\right]_{-}^{+}\right|_{\tilde{y}}=-\kappa_{5}^{2}\left(-\sigma_{\tilde{y}} \delta_{\alpha}^{\beta}+\left.T_{\alpha}^{\beta}\right|_{\tilde{y}}\right)
$$


- brane II

$$
\left.\left(K_{\alpha}^{\beta}-\delta_{\alpha}^{\beta} K\right)\right|_{y_{0}}=\frac{\kappa_{5}^{2}}{2}\left(-\sigma_{y_{0}} \delta_{\alpha}^{\beta}+\left.T_{\alpha}^{\beta}\right|_{y_{0}}\right)
$$

where the factor $1 / 2$ appears on the right-hand-side of Eqs. (2.16) and (2.18) because we have $\mathrm{Z}_{2}$-symmetry at the boundary branes. We therefore assume the tension on brane I to be positive $\left(\sigma_{0}>0\right)$ and the tension on brane II to be negative $\left(\sigma_{y_{0}}<0\right)$.

At the lowest order, matter is neglected and as in the two branes case the only equation to solve is the equation for $K_{\alpha}^{\beta}$, as $E_{\alpha}^{\beta}$ is taken to be zero at this order. In the bulk and at this order, the Lie derivative for $K_{\alpha}^{\beta}$ reads [8]

$$
\mathcal{L}_{n} K_{\alpha}^{\beta(0)}=e^{-\phi} \partial_{y} K_{\alpha}^{\beta(0)}=\frac{1}{\ell^{2}} \delta_{\alpha}^{\beta}-K_{\alpha}^{\gamma(0)} K_{\gamma}^{\beta(0)}
$$

In each AdS slice equation (2.19) has solution [8]

$$
K_{\alpha}^{\beta(0)}=-\frac{1}{\ell} \delta_{\alpha}^{\beta}
$$

where $\ell$ is now either $\ell_{1}$ or $\ell_{2}$ depending on which AdS slice is under consideration. Moreover, from the definition of the extrinsic curvature (2.11), in each AdS region the metric at zero order reads 8

$$
q_{\alpha \beta}^{(0)}(y, x)=e^{-2 d(y, x) / \ell} h_{\alpha \beta}(x)
$$

where

$$
d \equiv \int_{\bar{y}}^{y} e^{\phi\left(y^{\prime}, x\right)} d y^{\prime}
$$

is the proper distance between $\bar{y}$, any fixed point on the extra-coordinate axis, and $y$, both points being in the same AdS region, and $h_{\alpha \beta}(x)$ is a tensor field which does not depend on the extra coordinate $y$ (but will in general depend on the coordinates on some hypersurface orthogonal to the extra-coordinate, and in particular on the coordinates on the branes).

In our generalised Randall-Sundrum type scenario equations (2.20), (2.16) and (2.18) imply

$$
K_{\alpha}^{\beta}=\left\{\begin{array}{cc}
-\frac{\kappa_{5}^{2}}{6} \sigma_{0} \delta_{\alpha}^{\beta} & 0<y<\tilde{y}, \\
\frac{\kappa_{5}^{2}}{6} \sigma_{y_{0}} \delta_{\alpha}^{\beta} & \tilde{y}<y<y_{0}
\end{array}\right.
$$

Furthermore for the background solution we can write

$$
\left.\left[K_{\alpha}^{\beta}-\delta_{\alpha}^{\beta} K\right]_{-}^{+}\right|_{\tilde{y}}=\left.\left[K_{\alpha}^{\beta}-\delta_{\alpha}^{\beta} K\right]_{-}^{+}\right|_{y_{0}}-\left.\left[K_{\alpha}^{\beta}-\delta_{\alpha}^{\beta} K\right]_{-}^{+}\right|_{0}
$$

and we then conclude, using solution (2.23), that there exits a fine tuning condition constraining the tension on brane III, namely [18]

$$
\sigma_{0}+\sigma_{y_{0}}+2 \sigma_{\tilde{y}}=0 .
$$

Finally, again from solution (2.20), the condition (2.25) can be expressed in terms of the AdS curvature scales $\ell_{1,2}$ as

$$
\sigma_{\tilde{y}}=\frac{3}{\kappa_{5}^{2}} \frac{\left(\ell_{1}-\ell_{2}\right)}{\ell_{1} \ell_{2}} .
$$

where for simplicity we will assume $\sigma_{\tilde{y}}>0$ and hence $\ell_{1}>\ell_{2}$. 


\subsection{First-order effective theory}

At first order and in the bulk, the Lie derivatives for the Weyl tensor and the extrinsic curvature read [8]

$$
\begin{aligned}
\mathcal{L}_{n} E_{\alpha \beta}^{(1)} & =\frac{2}{\ell} E_{\alpha \beta}^{(1)} \\
\mathcal{L}_{n} K_{\alpha}^{\beta(1)} & =-\left(D_{\alpha} D^{\beta} \phi+D_{\alpha} \phi D^{\beta} \phi\right)+\frac{2}{\ell} K_{\alpha}^{\beta(1)}-E_{\alpha}^{\beta(1)}
\end{aligned}
$$

where $D^{\alpha}$ is the covariant derivative with respect to the induced metric $q_{\mu \nu}(y)$. These firstorder evolution equations have solutions in each AdS slice [8]

$$
E_{\alpha}^{\beta(1)}(y, x)=e^{4 d / \ell} E_{\alpha}^{\beta(1)}(\bar{y}, x)
$$

where the zero-order induced metric (2.21) has been used to raise the indices, and [8]

$$
\begin{aligned}
K_{\alpha}^{\beta(1)}(y, x) & =e^{2 d / \ell} K_{\alpha}^{\beta(1)}(\bar{y}, x)-\frac{\ell}{2}\left[1-e^{-2 d / \ell}\right] E_{\alpha}^{\beta(1)}(y, x) \\
& -\left[D_{\alpha} D^{\beta} d-\frac{1}{\ell}\left(D_{\alpha} d D^{\beta} d-\frac{1}{2} \delta_{\alpha}^{\beta}(D d)^{2}\right)\right]
\end{aligned}
$$

where again $d$ is the proper distance (2.22) between a generic point $\bar{y}$ on the extra-coordinate axis and $y$.

In the following we drop the first-order superscripts, assuming that all the quantities are evaluated at first order.

We define

$$
\left.\bar{K}_{\alpha}^{\beta}\right|_{y} \equiv K_{\alpha}^{\beta}(y, x)-\delta_{\alpha}^{\beta} K(y, x) .
$$

From equation (2.30), and integrating from brane I to brane III, we have

$$
\begin{aligned}
\left.\bar{K}_{\alpha}^{\beta}\right|_{\tilde{y}} & =e^{2 d_{1} / \ell_{1}}\left(-\left.\frac{\kappa_{5}^{2}}{2} T_{\alpha}^{\beta}\right|_{0}\right)-\left.\frac{\ell_{1}}{2}\left(1-e^{-2 d_{1} / \ell_{1}}\right) E_{\alpha}^{\beta}\right|_{\tilde{y}} ^{-} \\
& -\left(D_{\alpha} D^{\beta}-\delta_{\alpha}^{\beta} D^{2}\right) d_{1}+\frac{1}{\ell_{1}}\left(D_{\alpha} d_{1} D^{\beta} d_{1}+\frac{1}{2} \delta_{\alpha}^{\beta}\left(D d_{1}\right)^{2}\right)
\end{aligned}
$$

where we have used equation (2.16). On the other side of brane III, but integrating back from brane II, we have

$$
\begin{aligned}
\left.\bar{K}_{\alpha}^{\beta}\right|_{\tilde{y}} ^{+} & =e^{-2 d_{2} / \ell_{2}}\left(\left.\frac{\kappa_{5}^{2}}{2} T_{\alpha}^{\beta}\right|_{y_{0}}\right)-\left.\frac{\ell_{2}}{2}\left(1-e^{2 d_{2} / \ell_{2}}\right) E_{\alpha}^{\beta}\right|_{\tilde{y}} ^{+} \\
& +\left(D_{\alpha} D^{\beta}-\delta_{\alpha}^{\beta} D^{2}\right) d_{2}+\frac{1}{\ell_{2}}\left(D_{\alpha} d_{2} D^{\beta} d_{2}+\frac{1}{2} \delta_{\alpha}^{\beta}\left(D d_{2}\right)^{2}\right)
\end{aligned}
$$

where this time we have used equation (2.18). Note that in Eqs. (2.32) and (2.33) $D^{\alpha}$ is the covariant derivative induced on brane III.

Due to the junction conditions for $K_{\alpha}^{\beta}$ on the two sides of brane III, $E_{\alpha}^{\beta}$ does not evolve continuously from brane I to brane II, unlike the two brane scenario where (2.29) can be 
applied everywhere in the bulk. In fact, continuity of the induced metric implies that the induced Einstein tensor should also be continuous

$$
\left.G_{\alpha}^{\beta}\right|_{\tilde{y}} ^{+}=\left.G_{\alpha}^{\beta}\right|_{\tilde{y}} ^{-}
$$

At first order Eq. (2.15) thus gives a junction condition for the projected Weyl tensor across the bulk brane

$$
\left.E_{\alpha}^{\beta}\right|_{\tilde{y}} ^{+}-\left.E_{\alpha}^{\beta}\right|_{\tilde{y}} ^{-}=-\left.\frac{2}{\ell_{2}} \bar{K}_{\alpha}^{\beta}\right|_{\tilde{y}} ^{+}+\left.\frac{2}{\ell_{1}} \bar{K}_{\alpha}^{\beta}\right|_{\tilde{y}}
$$

In order to obtain an explicit expression for $\left.E_{\alpha}^{\beta}\right|_{\tilde{y}}$ on either side of brane III, we extract from equations (2.17), (2.32), (2.33) and (2.35) a set of two independent equations for the unknowns $\left.E_{\alpha}^{\beta}\right|_{\tilde{y}} ^{+}$and $\left.E_{\alpha}^{\beta}\right|_{\tilde{y}} ^{-}$, which can then be solved. From (2.17), (2.32) and (2.33) we obtain a first equation for $\left.E_{\alpha}^{\beta}\right|_{\tilde{y}} ^{+}$and $\left.E_{\alpha}^{\beta}\right|_{\tilde{y}} ^{-}$,

$$
\begin{aligned}
\left.\frac{\ell_{2}}{2} \mathcal{A} E_{\alpha}^{\beta}\right|_{\tilde{y}} ^{+}-\left.\frac{\ell_{1}}{2} \mathcal{B} E_{\alpha}^{\beta}\right|_{\tilde{y}} & =\frac{\kappa_{5}^{2}}{2}\left(\left.2 T_{\alpha}^{\beta}\right|_{\tilde{y}}+\left.e^{-2 d_{2} / \ell_{2}} T_{\alpha}^{\beta}\right|_{y_{0}}+\left.e^{2 d_{1} / \ell_{1}} T_{\alpha}^{\beta}\right|_{0}\right) \\
& +\left(D_{\alpha} D^{\beta}-\delta_{\alpha}^{\beta} D^{2}\right)\left(d_{2}+d_{1}\right) \\
+\frac{1}{\ell_{2}}\left(D_{\alpha} d_{2} D^{\beta} d_{2}+\frac{1}{2} \delta_{\alpha}^{\beta}\left(D d_{2}\right)^{2}\right) & -\frac{1}{\ell_{1}}\left(D_{\alpha} d_{1} D^{\beta} d_{1}+\frac{1}{2} \delta_{\alpha}^{\beta}\left(D d_{1}\right)^{2}\right)
\end{aligned}
$$

where

$$
\begin{aligned}
\mathcal{A} & =\left(1-e^{2 d_{2} / \ell_{2}}\right) \\
\mathcal{B} & =\left(1-e^{-2 d_{1} / \ell_{1}}\right)
\end{aligned}
$$

The second independent equation for $\left.E_{\alpha}^{\beta}\right|_{\tilde{y}} ^{+}$and $\left.E_{\alpha}^{\beta}\right|_{\tilde{y}} ^{-}$is directly obtained from (2.35) and equations (2.32), (2.33)

$$
\begin{aligned}
\left.E_{\alpha}^{\beta}\right|_{\tilde{y}} ^{+}-\left.E_{\alpha}^{\beta}\right|_{\tilde{y}} ^{-} & =\left.\left(1-e^{2 d_{2} / \ell_{2}}\right) E_{\alpha}^{\beta}\right|_{\tilde{y}} ^{+} \\
& -\left.\left(1-e^{-2 d_{1} / \ell_{1}}\right) E_{\alpha}^{\beta}\right|_{\tilde{y}} ^{-}-\frac{\kappa_{5}^{2}}{\ell_{2}}\left(\left.e^{-2 d_{2} / \ell_{2}} T_{\alpha}^{\beta}\right|_{y_{0}}\right) \\
& -\frac{\kappa_{5}^{2}}{\ell_{1}}\left(\left.e^{2 d_{1} / \ell_{1}} T_{\alpha}^{\beta}\right|_{0}\right)-2\left(D_{\alpha} D^{\beta}-\delta_{\alpha}^{\beta} D^{2}\right)\left(\frac{d_{2}}{\ell_{2}}+\frac{d_{1}}{\ell_{1}}\right) \\
& -2\left(D_{\alpha} \frac{d_{2}}{\ell_{2}} D^{\beta} \frac{d_{2}}{\ell_{2}}+\frac{1}{2} \delta_{\alpha}^{\beta}\left(D \frac{d_{2}}{\ell_{2}}\right)^{2}\right) \\
& +2\left(D_{\alpha} \frac{d_{1}}{\ell_{1}} D^{\beta} \frac{d_{1}}{\ell_{1}}+\frac{1}{2} \delta_{\alpha}^{\beta}\left(D \frac{d_{1}}{\ell_{1}}\right)^{2}\right)
\end{aligned}
$$

Equations (2.36) and (2.38) can be solved, for instance, for $\left.E_{\alpha}^{\beta}\right|_{\tilde{y}} ^{+}[12$, and the unique induced Einstein equation on the bulk brane are then derived, using the obtained expression for $\left.E_{\alpha}^{\beta}\right|_{\tilde{y}} ^{+}$in equation (2.15). After some rearrangements, we finally obtain

$$
\left.G_{\alpha}^{\beta}\right|_{\tilde{y}}=\frac{\kappa_{5}^{2}}{\ell_{3}}\left[\left.T_{\alpha}^{\beta}\right|_{\tilde{y}}+\left.\frac{e^{4 d_{1} / \ell_{1}}}{2} T_{\alpha}^{\beta}\right|_{0}\right]+\left.\frac{\kappa_{5}^{2}}{2 \ell_{3}} e^{-4 d_{2} / \ell_{2}} T_{\alpha}^{\beta}\right|_{y_{0}}
$$




$$
\begin{aligned}
& +\frac{e^{2 d_{1} / \ell_{1}}}{\ell_{3}}\left[D_{\alpha} D^{\beta}-\delta_{\alpha}^{\beta} D^{2}\right] d_{1}+\frac{e^{-2 d_{2} / \ell_{2}}}{\ell_{3}}\left[D_{\alpha} D^{\beta}-\delta_{\alpha}^{\beta} D^{2}\right] d_{2} \\
& -\frac{e^{2 d_{1} / \ell_{1}}}{\ell_{1} \ell_{3}}\left[D_{\alpha} d_{1} D^{\beta} d_{1}+\frac{1}{2} \delta_{\alpha}^{\beta} D^{2} d_{1}\right] \\
& +\frac{e^{-2 d_{2} / \ell_{2}}}{\ell_{2} \ell_{3}}\left[D_{\alpha} d_{2} D^{\beta} d_{2}+\frac{1}{2} \delta_{\alpha}^{\beta} D^{2} d_{2}\right]
\end{aligned}
$$

where $\ell_{3}$ is defined as follows

$$
\ell_{3} \equiv \frac{\ell_{1}}{2}\left(e^{2 d_{1} / \ell_{1}}-1\right)+\frac{\ell_{2}}{2}\left(1-e^{-2 d_{2} / \ell_{2}}\right)=\ell_{1} e^{d_{1} / \ell_{1}} \sinh \left(\frac{d_{1}}{\ell_{1}}\right)+\ell_{2} e^{-d_{2} / \ell_{2}} \sinh \left(\frac{d_{2}}{\ell_{2}}\right) .
$$

We now show explicitly that the effective theory at first order is indeed a generalised Brans-Dicke theory with two independent scalar fields. We may define a first dimensionless scalar field $\Phi$ to be

$$
\ell \Phi \equiv \ell_{3}
$$

where $\ell$ is an arbitrary unit of length and $\ell_{3}$ is given in (2.40), so that the kinetic terms for $d_{1}$ and $d_{2}$ in (2.39) can be rewritten as

$$
\frac{1}{\Phi}\left[D_{\alpha} D^{\beta}-\delta_{\alpha}^{\beta} D^{2}\right] \Phi+\mathcal{C}
$$

where

$$
\mathcal{C}=-\frac{3 e^{2 d_{1} / \ell_{1}}}{\ell_{1} \ell_{3}}\left[D_{\alpha} d_{1} D^{\beta} d_{1}-\frac{1}{2} \delta_{\alpha}^{\beta} D^{2} d_{1}\right]+\frac{3 e^{-2 d_{2} / \ell_{2}}}{\ell_{2} \ell_{3}}\left[D_{\alpha} d_{2} D^{\beta} d_{2}-\frac{1}{2} \delta_{\alpha}^{\beta} D^{2} d_{2}\right] .
$$

We can define a second scalar field, $\Psi$, which will have only first order derivatives appearing in Eq. (2.39) and should be as well a function of the two proper distances $d_{1}$ and $d_{2}$ (2.7). In general we can write

$$
\Psi \equiv \Psi(u)
$$

where $u=u\left(d_{1}, d_{2}\right)$ and hence

$$
\ell D_{\alpha} \Psi\left(u\left(d_{1}, d_{2}\right)\right)=\Psi_{1} D_{\alpha} d_{1}+\Psi_{2} D_{\alpha} d_{2}
$$

where $\Psi_{i} \equiv \ell \Psi^{\prime} \frac{\partial u}{\partial d_{i}}$ if $\Psi^{\prime}=\frac{d \Psi}{d u}$. Our objective is to be able to write $\mathcal{C}$ defined in equation (2.43) as

$$
\mathcal{C}=\frac{\omega(\Phi)}{\Phi^{2}}\left[\left(D_{\alpha} \Phi D^{\beta} \Phi-\frac{1}{2} \delta_{\alpha}^{\beta}(D \Phi)^{2}\right)-\Gamma(\Phi)\left(D_{\alpha} \Psi D^{\beta} \Psi-\frac{1}{2} \delta_{\alpha}^{\beta}(D \Psi)^{2}\right)\right] .
$$

Considering that in $\mathcal{C}$ there are no mixed terms $D_{\alpha} d_{1} D^{\beta} d_{2}$ or $D_{\alpha} d_{2} D^{\beta} d_{1}$, we get a first contraint on $\Gamma(\Phi)$ and $\Psi$, which reads

$$
e^{2 d_{1} / \ell_{1}-2 d_{2} / \ell_{2}}=\Gamma \Psi_{1} \Psi_{2}
$$

where $\Phi_{i} \equiv \ell\left(\partial \Phi / \partial d_{i}\right)$ and $\Psi_{i}$ are defined as before. 
A second constraint comes directly from the form of equation (2.45)

$$
\begin{aligned}
\ell^{2}\left[D_{\alpha} \Phi D^{\beta} \Phi-\Gamma\left(D_{\alpha} \Psi D^{\beta} \Psi\right)\right] & =\left[e^{4 d_{1} / \ell_{1}}-\Gamma \Psi_{1}^{2}\right] D_{\alpha} d_{1} D^{\beta} d_{1} \\
& +\left[e^{-4 d_{2} / \ell_{2}}-\Gamma \Psi_{2}^{2}\right] D_{\alpha} d_{2} D^{\beta} d_{2}
\end{aligned}
$$

Comparing the ratio of the coefficients in equations (2.43) and (2.47) then implies

$$
\frac{e^{4 d_{1} / \ell_{1}}-\Gamma \Psi_{1}^{2}}{e^{-4 d_{2} / \ell_{2}}-\Gamma \Psi_{2}^{2}}=-\frac{\ell_{2}}{\ell_{1}} e^{2 d_{1} / \ell_{1}+2 d_{2} / \ell_{2}} \quad .
$$

Using (2.46) to eliminate $\Gamma$ in (2.48), we obtain

$$
\left[1-\left(\frac{\Psi_{1}}{\Psi_{2}}\right) e^{-2 d_{1} / \ell_{1}-2 d_{2} / \ell_{2}}\right]\left[\ell_{1}-\ell_{2}\left(\frac{\Psi_{2}}{\Psi_{1}}\right)\right]=0 .
$$

The solutions of (2.49) are

$$
\frac{\Psi_{1}}{\Psi_{2}}=e^{2 d_{1} / \ell_{1}+2 d_{2} / \ell_{2}} ; \quad \frac{\Psi_{1}}{\Psi_{2}}=\frac{\ell_{2}}{\ell_{1}} .
$$

The first solution corresponds to the limit in which $\Phi=\Psi$ and both coefficients on the right hand side of (2.47) vanish. Choosing the second solution of (2.49) and using the defintion of $\Psi_{i}$, we obtain

$$
u=\frac{d_{1}}{\ell_{1}}+\frac{d_{2}}{\ell_{2}} .
$$

From the first constraint (2.46) and using $\Psi_{i}=\Psi^{\prime} \ell\left(\ell_{i}\right)^{-1}$ we obtain a differential equation for $\Psi(u)$ which reads

$$
\Psi^{\prime}=\frac{\sqrt{\ell_{1} \ell_{2}}}{\ell \sqrt{\Gamma}} e^{u} \frac{2 \ell \Phi+\ell_{1}-\ell_{2}}{\ell_{1} e^{2 u}-\ell_{2}} .
$$

where we have used (2.41).

In order for $\Psi$ to be only a function of $u$, and not of $\Phi$, as the scalar fields should be independent, we require that in equation (2.52)

$$
\ell^{2} \Gamma(\Phi)=\left(\ell \Phi+\frac{\ell_{1}}{2}-\frac{\ell_{2}}{2}\right)^{2} .
$$

Equation (2.52) then becomes

$$
\Psi^{\prime}=\frac{2 \sqrt{\ell_{1} \ell_{2}} e^{u}}{\ell_{1} e^{2 u}-\ell_{2}}
$$

and integrating we obtain

$$
\Psi(u)=\ln \left|\left(\frac{z-1}{z+1}\right)\right| \text { where } z=\sqrt{\frac{\ell_{1}}{\ell_{2}}} e^{u}
$$

so that

$$
e^{u}=\sqrt{\frac{\ell_{2}}{\ell_{1}}} \operatorname{coth}\left(\frac{\Psi}{2}\right)
$$


Imposing now (2.45), from both (2.46) and (2.48), we obtain

$$
\omega(\Phi)=-\frac{3}{2}\left(\frac{\ell \Phi}{\ell \Phi+\left(\frac{\ell_{1}}{2}-\frac{\ell_{2}}{2}\right)}\right) .
$$

Finally, the Einstein equations on brane III (2.39) can be written as

$$
\begin{aligned}
\left.G_{\alpha}^{\beta}\right|_{\tilde{y}} & =\left.\frac{\kappa_{5}^{2}}{\ell \Phi} T_{\alpha}^{\beta}\right|_{\tilde{y}}+\left.\frac{\kappa_{5}^{2}}{\Phi}\left(\frac{2 \ell \Gamma}{\ell_{1}^{2}}\right) \cosh ^{4}\left(\frac{\Psi}{2}\right) T_{\alpha}^{\beta}\right|_{0} \\
& +\left.\frac{\kappa_{5}^{2}}{\Phi}\left(\frac{2 \ell \Gamma}{\ell_{2}^{2}}\right) \sinh ^{4}\left(\frac{\Psi}{2}\right) T_{\alpha}^{\beta}\right|_{y_{0}}+\frac{1}{\Phi}\left[D_{\alpha} D^{\beta}-\delta_{\alpha}^{\beta} D^{2}\right] \Phi \\
& +\frac{\omega(\Phi)}{\Phi^{2}}\left[D_{\alpha} \Phi D^{\beta} \Phi-\frac{1}{2} \delta_{\alpha}^{\beta}(D \Phi)^{2}\right] \\
& -\frac{\omega(\Phi)}{\Phi^{2}} \Gamma(\Phi)\left[D_{\alpha} \Psi D^{\beta} \Psi-\frac{1}{2} \delta_{\alpha}^{\beta}(D \Psi)^{2}\right] .
\end{aligned}
$$

where the scalar fields $\Psi$ and $\Psi$ are defined as in Eqs. (2.41) and (2.55), and $\Gamma(\Phi)$ and $\omega(\Phi)$ are defined in Eqs. (2.53) and (2.57).

We can conclude from (2.58) that at low energy the effective theory on brane III is a generalized Brans-Dicke theory with two scalar fields, namely $\Psi$ and $\Phi$. This conclusion is a generalization of the result obtained in [6] and [8] for a two brane system. Note that although we find the Brans-Dicke parameter on the bulk brane, $\omega(\Phi)$, is negative, it is greater than $-3 / 2$ and thus there is no instability. This is most clearly seen when one writes the effective action in terms of scalar fields minimally coupled to the scalar curvature, i.e., in the Einstein frame. Also $\omega(\Phi) \Gamma(\Phi)$ is negative and thus the kinetic terms for the second field $\Psi$ are also are of the correct sign and again there is no instability.

\subsection{The Einstein frame}

In this last subsection, we show that the theory is, as expected for Brans-Dicke theories, conformally equivalent to Einstein gravity with two scalar fields minimally coupled with respect to the metric.

The effective action which yields the field equations (2.58), when written in terms of the induced metric on brane III, reads

$$
\begin{aligned}
S_{\tilde{y}} & =\frac{\ell}{2 \kappa_{5}^{2}} \int d^{4} x \sqrt{-g}\left[\Phi R-\frac{\omega(\Phi)}{\Phi}\left[(\nabla \Phi)^{2}-\Gamma(\Phi)(\nabla \Psi)^{2}\right]\right] \\
& +\int d^{4} x \sqrt{-g}\left[\mathcal{L}(\tilde{y})+\mathcal{F}_{0}(\Phi, \Psi) \mathcal{L}(0)+\mathcal{F}_{y_{0}}(\Phi, \Psi) \mathcal{L}\left(y_{0}\right)\right]
\end{aligned}
$$

where

$$
\mathcal{F}_{0}=2 \frac{\ell^{2} \Gamma}{\ell_{1}^{2}} \cosh ^{4}\left(\frac{\Psi}{2}\right) ; \quad \mathcal{F}_{y_{0}}=2 \frac{\ell^{2} \Gamma}{\ell_{2}^{2}} \sinh ^{4}\left(\frac{\Psi}{2}\right)
$$


Performing a conformal transformation $\tilde{g}_{\alpha \beta}=\Omega^{2} g_{\alpha \beta}$, the effective action on brane III (2.59) becomes

$$
\begin{aligned}
\tilde{S}_{\tilde{y}} & =\frac{1}{2 \kappa_{4}^{2}} \int d^{4} x \sqrt{-\tilde{g}}\left[\left[\tilde{R}-\frac{3}{2}\left(\frac{\tilde{\nabla} \Phi}{\Phi}\right)^{2}\right]-\omega(\Phi)\left[\left(\frac{\tilde{\nabla} \Phi}{\Phi}\right)^{2}-\Gamma(\Phi)\left(\frac{\tilde{\nabla} \Psi}{\Phi}\right)^{2}\right]\right] \\
& +\frac{\kappa_{5}^{4}}{\ell^{2} \kappa_{4}^{4}} \int d^{4} x \sqrt{-\tilde{g}} \Phi^{-2}\left[\mathcal{L}_{\tilde{y}}+\mathcal{F}_{0}(\Phi, \Psi) \mathcal{L}(0)+\mathcal{F}_{y_{0}}(\Phi, \Psi) \mathcal{L}\left(y_{0}\right)\right]
\end{aligned}
$$

where we chose

$$
\Phi \Omega^{-2}=\kappa_{5}^{2}\left(\ell \kappa_{4}^{2}\right)^{-1}
$$

and the Ricci scalar is now the Ricci scalar with respect to the conformally transformed metric. The constant $\kappa_{4}$ is an arbitrary constant which is related to the effective $4 D$ Newton's constant $G_{(4)}$ via $8 \pi G_{(4)}=\kappa_{4}^{2}$.

Defining $\gamma=2 \ell\left(\ell_{1}-\ell_{2}\right)^{-1}$ (which, given our choice $\ell_{1}>\ell_{2}$, is a positive constant) and taking into account (2.53) (2.57), action (2.61) becomes

$$
\begin{aligned}
\tilde{S}_{\tilde{y}} & =\frac{1}{2 \kappa_{4}^{2}} \int d^{4} x \sqrt{-\tilde{g}}\left[\tilde{R}-\frac{3}{2}\left[\frac{1}{(\gamma \Phi+1)}\left(\frac{\tilde{\nabla} \Phi}{\Phi}\right)^{2}+\tilde{\Gamma}(\Phi)(\tilde{\nabla} \Psi)^{2}\right]\right] \\
& +\frac{\kappa_{5}^{4}}{\ell^{2} \kappa_{4}^{4}} \int d^{4} x \sqrt{-\tilde{g}} \Phi^{-2}\left[\mathcal{L}_{\tilde{y}}+\mathcal{F}_{0}(\Phi, \Psi) \mathcal{L}(0)+\mathcal{F}_{y_{0}}(\Phi, \Psi) \mathcal{L}\left(y_{0}\right)\right]
\end{aligned}
$$

where $\tilde{\Gamma}(\Phi)=(\gamma \Phi+1)(\gamma \Phi)^{-1}$. We now define

$$
(\tilde{\nabla} \eta)^{2}=\frac{3}{2}\left[\frac{1}{\gamma \Phi+1}\right]\left(\frac{\tilde{\nabla} \Phi}{\Phi}\right)^{2}
$$

from which we get, as in the two brane case,

$$
\operatorname{coth}\left(\frac{\eta}{\sqrt{6}}\right)=\sqrt{\gamma \Phi+1}
$$

Finally given (2.65) we have

$$
\begin{aligned}
\tilde{S}_{\tilde{y}}= & \frac{1}{2 \kappa_{4}^{2}} \int d^{4} x \sqrt{-\tilde{g}}\left[\tilde{R}-(\tilde{\nabla} \eta)^{2}-\frac{3}{2} \cosh ^{2}\left(\frac{\eta}{\sqrt{6}}\right)(\tilde{\nabla} \Psi)^{2}\right] \\
& +\frac{\kappa_{5}^{4} \gamma^{2}}{\ell^{2} \kappa_{4}^{4}} \int d^{4} x \sqrt{-\tilde{g}} \sinh ^{4}\left(\frac{\eta}{\sqrt{6}}\right) \mathcal{L}_{\tilde{y}} \\
& +\frac{\kappa_{5}^{4} \gamma^{2}}{\kappa_{4}^{4}} \int d^{4} x \sqrt{-\tilde{g}} 2 \cosh ^{4}\left(\frac{\eta}{\sqrt{6}}\right)\left[\frac{\cosh ^{4}\left(\frac{\Psi}{2}\right)}{\ell_{1}^{2}} \mathcal{L}(0)+\frac{\sinh ^{4}\left(\frac{\Psi}{2}\right)}{\ell_{2}^{2}} \mathcal{L}\left(y_{0}\right)\right] .
\end{aligned}
$$




\section{Conclusions and Discussions}

In this paper we derived the low-energy effective theory for a generalized Randall-Sundrum scenario, with three $4 D$ branes embedded in a $5 D$ AdS bulk. Two of the branes are located at the fixed points of the orbifold, but the third brane can be located anywhere in between. By construction, the metrics on the three branes are all connected by an appropriate conformal transformation. It is therefore enough to derive the $4 D$ effective Einstein equations on the third brane, as the effective theory on the other branes can then be obtained performing a conformal transformation.

We followed the covariant approach adopted in Ref. [8] to derive the low-energy effective theory for a two brane Randall-Sundrum system. We considered an expansion of the extrinsic curvature and of the projected Weyl tensor, where the expansion parameter is the ratio of the energy density on the brane to the vacuum energy density as in Refs. [6, 8]. In each separate region of the AdS bulk, the evolution equations for the extrinsic curvature tensor and for the projected Weyl tensor are, at each order, the same as in the two brane scenario and solutions to the first-order equations can be found separately in each AdS slice.

The presence of the third brane obliges the evolution of the projected Weyl tensor to be discontinous. From the requirement of consistency of the Einstein equations and from the junction conditions for the extrinsic curvature tensor at the third brane we obtain a junction condition for the projected Weyl tensor in terms of the extrinsic curvatures on both sides of the branes and the sources on both branes. Once an expression for the Weyl tensor as a function of the sources on the branes was obtained, we have finally derived the first order $4 D$ effective Einstein equations on the third brane. The resulting theory is a generalized Brans-Dicke theory with two independent scalar fields. The appearance of two independent scalar fields is not surprising as the three brane scenario is characterized by two natural scalar degrees of freedom: the overall size of the orbifold and the position of the third brane. A non-minimal coupling of the fields is found with respect to matter on the other two branes. We have then showed that the effective theory is conformally equivalent to Einstein gravity plus two scalar fields minimally coupled with the geometry.

We can conclude that the interpretation of the radion field in the two brane scenario can be generalized to a three-brane scenario in which there exists an additional scalar degree of freedom. In the two brane case the realization at first order of the non-local Einstein gravity, with the generalized dark radiation term, as a local effective theory is described by the radion field which appears in the equations through its derivatives. In the three brane case, as in the case where a scalar bulk field is living in the bulk [19, 20], two scalar fields both contribute to the realization of the (local) effective theory on the brane.

A moving bulk brane of the sort described here was discussed as a simple realisation of the original ekpyrotic scenario [10] where the collision of the bulk brane with a Minkowski boundary brane was interpreted as initiating a hot big bang cosmology on the brane. (A moving bulk brane has also been studied in M-theory effective action [21].) Unlike the collision of two boundary branes in the later cyclic model [22], the bulk spacetime does not disappear at the collision of a bulk brane with the boundary and hence the outgoing state is completely determined by the incoming state [17]. If the boundary brane tension does not obey the RS fine-tuning (1.1) then it may be inflating before the collision and the possibility

that a collision of the bulk brane with the boundary may trigger the end of inflation was 
studied in Ref. [14], using the effective action derived in this paper. It remains to be seen whether the techniques described in this paper might be suitable for deriving a low-energy effective action capable of incorporating the gravitational back-reaction of moving branes in flux-compactification scenarios, see, e.g., Refs. [13, 23].

\section{Acknowledgements}

LSR acknowledges support from the Swiss National Science Foundation, grant number 205320-103833/1. DW is supported in part by PPARC grant number PPA/G/S/2002/00576.

\section{References}

[1] A. Lukas, B. A. Ovrut and D. Waldram, Phys. Rev. D 60, 086001 (1999) arXiv:hep-th/9806022]; A. Lukas, B. A. Ovrut and D. Waldram, Nucl. Phys. B 552, 246 (1999) [arXiv:hep-th/9806051]; M. Brandle, A. Lukas and B. A. Ovrut, Phys. Rev. D 63, 026003 (2000) [arXiv:hep-th/0003256].

[2] L. Randall and R. Sundrum, Phys. Rev. Lett. 83, 3370 (1999) arXiv:hep-ph/9905221.

[3] L. Randall and R. Sundrum, Phys. Rev. Lett. 83, 4690 (1999) arXiv:hep-th/9906064.

[4] T. Shiromizu, K. Maeda and M. Sasaki, Phys. Rev. D 62, 024012 (2000) arXiv:gr-qc/9910076.

[5] R. Maartens, Phys. Rev. D 62, 084023 (2000) arXiv:hep-th/0004166; R. Maartens, arXiv:gr-qc/0101059, R. Maartens, Living Rev. Relativity 7, 7 (2004) arXiv:gr-qc/0312059.

[6] S. Kanno and J. Soda, Phys. Rev. D 66, 043526 (2002) arXiv:hep-th/0205188];

S. Kanno and J. Soda, Phys. Rev. D 66, 083506 (2002) arXiv:hep-th/0207029|.

[7] T. Wiseman, Class. Quant. Grav. 19, 3083 (2002) arXiv:hep-th/0201127.

[8] T. Shiromizu and K. Koyama, Phys. Rev. D 67, 084022 (2003) arXiv:hep-th/0210066.

[9] S. Kanno and J. Soda, Astrophys. Space Sci. 283, 481 (2003) arXiv:gr-qc/0209087.

[10] J. Khoury, B. A. Ovrut, P. J. Steinhardt and N. Turok, Phys. Rev. D 64, 123522 (2001) arXiv:hep-th/0103239; A. J. Tolley, N. Turok and P. J. Steinhardt, Phys. Rev. D 69, 106005 (2004) |arXiv:hep-th/0306109].

[11] S. Kanno, M. Sasaki and J. Soda, Prog. Theor. Phys. 109, 357 (2003) arXiv:hep-th/0210250.

[12] L. Cotta-Ramusino, MPhil thesis, Low energy effective theory for brane world models, Portsmouth University, 2004. 
[13] K. Koyama and K. Koyama, Class. Quant. Grav. 22, 3431 (2005) arXiv:hep-th/0505256.

[14] S. Kanno, J. Soda and D. Wands, JCAP 0508, 002 (2005) arXiv:hep-th/0506167.

[15] C. de Rham, S. Fujii, T. Shiromizu and H. Yoshino, Phys. Rev. D 72, 123522 (2005) arXiv:hep-th/0509194.

[16] I. I. Kogan, S. Mouslopoulos, A. Papazoglou and L. Pilo, Nucl. Phys. B 625, 179 (2002) arXiv:hep-th/0105255.

[17] D. Langlois, K. Maeda and D. Wands, Phys. Rev. Lett. 88, 181301 (2002) arXiv:gr-qc/0111013.

[18] G. W. Gibbons, R. Kallosh and A. D. Linde, JHEP 0101, 022 (2001) arXiv:hep-th/0011225.

[19] G. A. Palma and A. C. Davis, Phys. Rev. D 70, 064021 (2004) arXiv:hep-th/0406091;

G. A. Palma and A. C. Davis, Phys. Rev. D 70, 106003 (2004) arXiv:hep-th/0407036.

[20] S. Kanno and J. Soda, Gen. Rel. Grav. 36, 689 (2004) arXiv:hep-th/0303203.

[21] E. J. Copeland, J. Gray and A. Lukas, Phys. Rev. D 64, 126003 (2001) arXiv:hep-th/0106285]; E. J. Copeland, J. Gray, A. Lukas and D. Skinner, Phys. Rev. D 66, 124007 (2002) |arXiv:hep-th/0207281].

[22] P. J. Steinhardt and N. Turok, arXiv:hep-th/0111030.

[23] D. Baumann, A. Dymarsky, I. R. Klebanov, J. Maldacena, L. McAllister and A. Murugan, arXiv:hep-th/0607050. 